IX.

Arbeiten aus dem Laboratorium für experimentelle Pharmakologie zu Strassburg.

\title{
135. Ueber Dinrese.
}

Zweiter Theil. 1)

Die Wirkung artificieller Bluteindickung auf Harn. absonderung und Lymphorrhöe.

Ein Beitrag zur Pharmakologie colloider Substanzen.

Von

Dr. phil. et med. Spiro,

Privatdocent und I. Assistent am physiol. chem. Inst.

Die nachfolgenden Untersuchungen knüpfen an ältere des Verfassers an 2), welche die eigenthümliche Wirkungsweise des Witte'sehen "Peptons" auf den Lymphfluss zum Gegenstand hatten, und haben aus den nachfolgenden Gründen sich zum Ziel gesetzt, die Einwirkung einer intravenösen Injection colloidaler Körper auf Harn und Lymphe zu untersuchen.

Bekanntlich batte zuerst Heidenhain in seiner die Forsehung so mächtig anregenden Arbeit: "Versuche und Fragen zur Lehre von der Lymphbildung:" 3) zwei Reihen von Mitteln, den Lymphfluss zu beschleunigen und zu modificiren, kennen gelehrt: die sogenannten Lymphagoga erster und zweiter Ordnung. Während für das Verständniss der letzteren eine Reihe von wichtigen Arbeiten seither beigebracht ist ${ }^{4}$ ), fehlt uns zur Erklärung der ersteren trotz maneher andeutender Fingerzeige noch ein wesentlicher Theil: Die Wirkungsweise der Lymphagoga zweiter Ordnung können wir wohl im wesent-

1) Vgl. Archiv f. experiment. Pathol. u. Pharmakol. Bd. XXXVIII, S. 369, 1897.

2) Archiv f. experiment. Pathol. u. Pharmakol. Bd. XXXVIII, S. 113, 1897.

3) Pflüger's Archiv Bd. XLIX, S. 209, 1891.

4) Vgl. namentlich die Arbeiten von W. Cohnstein, eine Uebersicht ron demselben: Pflüger's Archiv Bd. LXIII, S. 587, 1896 und Lubarsch-Ostertag's Ergebnisse Bd. III, S. 563, 1896. 
lichen jetzt als eine molecular-chemische, als eine "Salzwirkung" im Sinne Schmiedeberg's 1 ) bezeichnen; hierauf deutet auch das Gesammtbild, das nach ihrer Injection zur Erscheinung kommt, vor allem, dass die Lymphorrböe nicht die einzige Wirkung ist, die der intravenösen Injection folgt, sondern dass, wie schon Heidenhain hervorhob, gleichzeitig mit der Lymphsecretion auch die Harnbildung angeregt wird. Inwieweit die Beobachtungen an Harn und Lymphe, für die, namentlich durch die Arbeiten von v. Li mbee ${ }^{2}$ ) und M inze ${ }^{3}$ ), sorgfältiges und werthvolles Material vorliegt, zu einer einheitlichen Enklärung des Gesammtwirkungsbildes ausreichen, soll an anderer Stelle auf Grund eigener ergänzender Untersuchungen dargethan werden; gerade aber bei der wesentlichen Uebereinstimmung, die die Salzwirkung in Bezug auf Harn und Lymphe zeigt, mussten auch die Lymphagoga erster Ordnung in ihrer diuretischen Wirkung genaner analysirt werden.

Für die Albumosen ist es bekannt, dass sie, trotzdem sie zum Theil im Harn wiedererscheinen, eine harntreibende Wirkung nicht haben, in grösseren Mengen sogar die Harnabsonderung unterdrü cken können. Da Heidenhain eine ähnliche Wirkung wie bei "Pepton" ausser bei Anwendung gewisser Extracte nur noch nach Injection von verdünntem Hühnereiweiss - und hier durchaus nieht regelmässig - beobachtet hat, so war zu vermuthen, dass hier nicht physikalische Eigenschaften der Präparate die Wirkung veranlassten, sondern vielleicht eigenthümliche, in ihnen enthaltene "specifische", fermentartig wirkende Körper. Durch Vergleich mit anderen colloidalen Körpern sollte also gezeigt werden, inwieweit bei der Einwirkung der Albumosen auf Lymphe und Harn neben etwaigen physikalischchemischen Eigenschaften der injicirten Körper auch specifische in Betracht kommen, und ob dureh diese Untersuchungen eine Erklärung für die so verschiedene Wirkungsweise auf Harn- und Lymphapparat zu erzielen wäre.

Einer kurzen Erörterung bedarf es hier vorerst wohl noch, ob den colloidalen Körpern ähnlich wie den krystalloiden iuberhanpt eine Salzwirkung im physikalischen Sinne zukommen kann; dass sie auch osmotischen Druck auszuüben im Stande sind, das haben vor vielen Jahren schon Pfeffer in seinen grundlegenden "osmo-

1) Grundriss der Arzneimittellehre 1895, S. 242.

2) Archiv f. experiment. Pathol. n. Pharmakol. Bd. XXV, S. 1, 1889.

3) Ebenda Bd. XLI, S. 74, 1898; vgl. auch die anderen Arbeiten aus der Reihe: "Zur Lehre von der Wirkang der Salze" von Hofmeister und seinen Schülern in diesem Archiv. 
tischen Untersuchungen" 1) und erst neuerdings wieder Starling" and Cohnstein ${ }^{3}$ ) nachgewiesen, ja man kann, wie joh jüngst gezeigt zu haben glaube ${ }^{4}$, die zwei "verschiedenen Welten der Materie", Krystalloide und Colloide, unter einem einheitlichen Gesichtspunkt zusammenfassen als Stoffe, die sich in ihrem physikalischen Gesammtverhalten nur quantitativ, nicht qualitativ von einander unterscheiden. Auch die folgenden Untersuchungen werden dies darthun.

Die Mäglichkeit, Versuche über künstliche Bluteindickung anzustellen, war gegeben durch die im Jahre 1894 erschienene Arbeit von Adalbert Czerny5). Dieser Autor zeigte, dass intravenöse Gaben colloider Körper von Hunden, Katzen und Kaninchen relativ gut vertragen werden. So vertragen nach ihm diese Thiere bis zu $0,4 \mathrm{~g}$ Gelatine und bis zu 0,46 g Gummi intravenös pro $100 \mathrm{~g}$ Körpergewicht, womit meine eigenen Trfahrungen ganz genau ubereinstimmen.

Ioh selbst stellte meine Versuche mit Gelatine and Gummi arabicum an, nur e in en Versuch habe ich mit krystallisirtem Serumalbumin (dargestellt nach $G$ ürber) gemacht, der jedoch keine Besonderheiten aufwies und in Bezug auf Harn- und Lymphwirkung nichts Eigenthümliches erkennen liess : namentlich eine Lymphwirkung wie bei den Lymphagogis erster Ordnung vermisste ich. Als Gelatine wurde die reinste käufliche des Handels verwandt, ebenso vom Gummi arabicum, dessen Lösung mit Soda vorsichtig neutralisirt wurde. Der Gehalt der Lösung an organischer Substanz wurde durch Troeknen und Wägung bestimmt, die Asche der Gummilösung (nicht ganz 1 Proc.) bestand aus kohlensaurem Kalk.

Da man bei diuretischen Versuchen an Kaninchen, um vergleichbare Harnquantitäten für kurze Zeiträume zu erhalten, mit Thieren zu experimentiren gewohnt ist, die 24-40 Stunden gehungert hatten, stellte ich meine ersten Versuche auch an solchen Hungerthieren an. Hier zeigten Injectionen von Gummi und Gelatine gar keine Einwirkung. Ioh wähle zur Illustrirung folgendes Protokoll.

\section{Versuch I.}

Kaninchen $2200 \mathrm{~g}$, hat 36 Stunden gehungert. Urethan-Narkose, Blasencanüle.

1) Leipzig 1877. S. 73.

2) Journal of Physiology Bd. XIX, p. 321, 1896.

3) Pflüger's Archiv Bd. LXIII, S. 607, 1896.

4) Ueber physikalische und physiologische Selection. Strassburg 1897.

5) Archiv f. experiment. Pathol. u. Pharmakol. Bd. XXXIV, S. 268. 
Zeit

9 h. $50 \mathrm{~m} .-10 \mathrm{~h} .-\mathrm{m}$.

10 h. - m. $-10 \mathrm{~h} .10 \mathrm{~m}$.

10 h. 10 m. -10 h. $20 \mathrm{~m}$.

10 h. $20 \mathrm{~m} .-10$ h. $50 \mathrm{~m}$. werden ganz allmählich $6 \mathrm{~g}$ Gummi arabicum, in $25 \mathrm{ccm}$ Wasser gelöst in die Vena jugularis infundirt.

10 h. 20 m. -10 h. 30 m.

$10 \mathrm{~h} .30 \mathrm{~m} .-10 \mathrm{~h} .40 \mathrm{~m}$.

10 h. 40 m. -10 h. 50 m.

$10 \mathrm{~h} .50 \mathrm{~m} .-11 \mathrm{~h} .-\mathrm{m}$.
Harnmenge

$0,15 \mathrm{cem}$

$0,1=$

$0,1=$

Auch eine erneute Injection von $2 g$ Gummi arabicum war (gemessen 11 h. - 11 h. 40 m.) ganz wirkungslos.

Während somit in Bezug auf die Diurese an Hungerthieren nur ein negatives Resultat erzielt wurde, das jedoch, wie weiter unten gezeigt werden soll, nicht unwichtig ist, kam ich za einem ausgesprochenen Resultat in Bezug auf die Lymphwirkung. Ich habe drei derartige Versuche an Hunden angestellt mit übereinstimmendem Resultat, so dass ich nur einen ausführlich wiedergebe.

\section{Versuch II.}

Junger Hund von $10500 \mathrm{~g}$. Der Hund hatte 48 Strnden vorher gehungert. Operation beendet um $11 \mathrm{~h}$.

\section{Zeit}

11 h. 25 m. -11 h. $35 \mathrm{~m}$.

11 h. $35 \mathrm{~m} .-11$ h. $45 \mathrm{~m}$.

$11 \mathrm{~h} .45 \mathrm{~m} .-11 \mathrm{~h} .55 \mathrm{~m}$.

$11 \mathrm{~h} .55 \mathrm{~m} .-12 \mathrm{~h} .05 \mathrm{~m}$.

$12 \mathrm{~h} .05 \mathrm{~m} .-12 \mathrm{~h} .15 \mathrm{~m}$.

$12 \mathrm{~h} .15 \mathrm{~m} .-12 \mathrm{~h} .25 \mathrm{~m}$.

12 h. $15 \mathrm{~m} .-12 \mathrm{~h} .25 \mathrm{~m}$.

$12 \mathrm{~h} .25 \mathrm{~m} .-12 \mathrm{~h} .35 \mathrm{~m}$.

12 h. $35 \mathrm{~m} .-12$ h. $45 \mathrm{~m}$.

12 h. 45 m. -12 h. $55 \mathrm{~m}$.

$12 \mathrm{~h} .55 \mathrm{~m} .-1 \mathrm{~h} .05 \mathrm{~m}$.
Lymphmenge aus dem.

Ductus Thoracicus

$$
\begin{aligned}
& 2,4 \text { cem } \\
& 2,2= \\
& 2,5= \\
& 1,9=\quad \text { Gerinnsel! } \\
& 2,1=
\end{aligned}
$$

Injection von $20 \mathrm{~g}$ Gummi arabicum (neutralisirt) in die Vena femoralis sinistra.

$1,8 \mathrm{ccm}$

$1,5=$

$1,2=$

$1,4=$

$1,3=$

Eine zu anderen Zwecken sich daran schliessende Peptoninfusion bewirkt starken Lymphfluss.

Dieses selbe Resultat ist, wie ich aus der inzwischen erschienenen ausführlichen Zusammenstellung von Cohnste in ersehe, auch von diesem selbst (mit Dr. P. Meissner) ${ }^{1}$ ) und von Lazarus Barlow ${ }^{2}$ ) erhoben worden. Man sieht also, dass im allgemeinen die colloidalen Körper auf die Lymphe nicht wie Pepton wirken. Dieser Befund

1) 1. c. S. 590 .

2) Journ. of Physiology Bd, XIX, p. 418, 1896. 
ist wohl einfach in physikalischer Weise so, wie es auch Cohnstein schon gethan hat, zu deuten: offenbar wirken die colloidalen Körper innerhalb des Gefässsystemes stark wasseranziehend, der Wassergehalt des Blutes nimmt, wie dies schon $\mathrm{Czerny}$ aus seinen Blutkörperchenzählungen geschIossen hat, stark zu, und die Gewebsflüssigkeit, die Lymphe, nimmt an Menge ab. Hiermit stimmen anch zahlreiche Trockenbestimmungen, die ich gemacht habe, überein, die stets eine Zunahme im Wassergehalt des Blutes der Thiere ergaben. Ich habe Steigerungen im Wassergehait gesehen von 78 Proc. bis auf 90 Proc. und Hand in Hand damit Abnahmen im Stickstoffgehalt des Blutes von 2,6 Proc. bis auf 1,5 und 1,4 Proc.

Zu ganz anderen Resultaten gelangt man namentlich für die Diurese, wenn man nicht an Hungerthieren arbeitet, sondern an gat genährten Kaninchen, namentlich solchen, welche besonders wasserreiche Nabrung $z \mathfrak{u}$ sich genommen haben. An diesen Thieren erhält man bei der Injection colloidaler Lösungen eine je nach der eingeführten Quantität steigende Harnflut. Ieh möehte sofort bemerken, dass die auftretende Diurese in jeder Beziehung mit der Salzdiurese übereinstimmt. Dies wird am besten hervortreten, wenn ich ein Protokoll über die Salzdiurese, wie sie ähnlich Münzer vor kurzer Zeit in grösserer Zahl publicirt hat, meinen Protokollen beifüge. Ioh habe bei beiden Körperklassen die Versuche ähnlich wie Münzer angestellt, d. h. um vergleichbare Zahlen zu erhalten, dem Thier in abgemessenen Zeiträumen abgemessene Quantitäten, z. B. etwa jede Minute 1 oder $2 \mathrm{ccm}$ der zu untersuchenden Lösung infundiren lassen.

\section{Versuch III.}

Kaninchen. $2000 \mathrm{~g} .3 \mathrm{~g}$ Urethan, Blasencanüle. Injectionsflüssigkeit: Leimlösung von 3 Proc.

\section{Zeit}

4 h. $05 \mathrm{~m} .-4 \mathrm{~h} .15 \mathrm{~m}$.

4 h. $15 \mathrm{~m} .-4$ h. $25 \mathrm{~m}$.

4 h. $25 \mathrm{~m} .-4 \mathrm{~h} .35 \mathrm{~m}$.

Von 4 h. $35 \mathrm{~m} .-4$ h. $45 \mathrm{~m}$.

4 h. $45 \mathrm{~m} .-4$ h. $55 \mathrm{~m}$.

$4 \mathrm{~h} .55 \mathrm{~m},-5 \mathrm{~h} .05 \mathrm{~m}$.

5 h. $05 \mathrm{~m} .-5$ h. $15 \mathrm{~m}$.

5 h. $15 \mathrm{~m} .-5$ h. $25 \mathrm{~m}$.

5 h. 25 m. -5 h. $35 \mathrm{~m}$.

5 h. $35 \mathrm{~m} .-5$ h. $45 \mathrm{~m}$.

5 h. $45 \mathrm{~m} .-5$ h. $55 \mathrm{~m}$.

5 h. $55 \mathrm{~m} .-6 \mathrm{~h} .05 \mathrm{~m}$.
Harnmenge

$0,6 \mathrm{ccm}$

$0,8=$

$0,6=$

alle Minuten je $2 \mathrm{~cm}$ der Lösung in die Vena jugularis injicirt.

$1,1 \mathrm{ccm}$

$1,5=$

$2,3=$

$3,7=$

$5,2=$

$6,2=$

$6,3=$

$3,7=$ 
Zeit

$6 \mathrm{~h} .05 \mathrm{~m} .-6 \mathrm{~h} .15 \mathrm{~m}$.

Harnmenge

$6 \mathrm{~h} .15 \mathrm{~m} .-6 \mathrm{~h} .25 \mathrm{~m}$.

$6 \mathrm{~h} .25 \mathrm{~m} .-6 \mathrm{~h} .35 \mathrm{~m}$.

$1,8 \mathrm{ccm}$

$0,6=$

$0,15=$

$6 \mathrm{~h} .25 \mathrm{~m}$. Athmung stark beschlennigt. $6 \mathrm{~h} .40 \mathrm{~m}$. Exitus.

Versuch IV.

Kaninchen, $3 \mathrm{~kg}$. Narkose mit 3,0 g Urethan. Blasencanüle. Zeit

8 h. 50 m. - 9 h. - m.

9 h. - m. -9 h. $10 \mathrm{~m}$.

9 h. 10 m. -9 h. $20 \mathrm{~m}$.

9 h. 20 m. -9 h. $30 \mathrm{~m}$.

Harnmenge

$0,2 \mathrm{ccm}$

$0,5=$

$0,8=$

$0,6=$

Von 9 h. $30 \mathrm{~m}$. ab alle Minuten je $2 \mathrm{ccm}$ einer 5,0 procentigen Kochsalzlösung intravenös.

9 h. $30 \mathrm{~m} .-9$ h. $40 \mathrm{~m}$.

9 h. $40 \mathrm{m.}-9 \mathrm{~h} .50 \mathrm{~m}$.

9 h. 50 m. -10 h. $-\mathrm{m}$.

10 h. - m. -10 h. $10 \mathrm{~m}$.

10 h. 10 m. -10 h. 20 m.

10 h. 20 m. -10 h. $30 \mathrm{~m}$.

10 h. $30 \mathrm{~m} .-10$ h. $40 \mathrm{~m}$.

10 h. $40 \mathrm{~m},-10$ h. $50 \mathrm{~m}$.

$10 \mathrm{~h} .50 \mathrm{~m} .-11 \mathrm{~h} .-\mathrm{m}$.

11 h. - m. -11 h. $10 \mathrm{~m}$.

11 h. $10 \mathrm{~m} .-11$ h. $20 \mathrm{~m}$.

11 h. $20 \mathrm{~m} .-11$ h. $30 \mathrm{~m}$.

11 h. $30 \mathrm{~m} .-11$ h. $40 \mathrm{~m}$.

6,8 cem
$15,6=$
$22=$
$33=$
$37=$
$27=$
$26=$
$19=$
$17=$
$13=$
$8=$
$3,2=$
$0,5=$

Von 11 h. 40 m. an Anurie, Krämpfe. 11 h. 57 m. Exitus.

Wie man aus den angeführten Zahlen ersieht, ist der Gang der Diurese in beiden Fällen ein ähnlicher: Er ist qualitatio durchaus nicht verschieden, sondern nur quantitativ. Während bei der Kochsalzdiurese bis zu 150 Proc. und mehr der eingeführten Flüssigkeitsquantitäten in einem entsprechenden Zeitabschnitt im Harn wiedererscheinen, kommen bei der Colloiddiurese böchstens 30-40 Proc. wieder zum Vorschein. Diese quantitative Differenz ist leicht zu erklären durch die Differenz der angewandten Molecülgrössen, und wenn auch der osmotische Druck, den colloidale Körper auszuüben im Stande sind, sehr klein ist, $d$ ass sie einen solchen im Thierkörper auszü̈ben vermögen, geht aus diesen Diurese. versuchen wohl unzweideutig hervor.

Die vollkommene Uebereinstimmung der Salz- und Colloiddiurese wird am anschaulichsten gezeigt durch eine Untersuchung des Blutes. Wie aus dem folgenden Versuchsprotokoll hervorgeht, zeigt das Blut nach Injection von Gummilösung z. B. ein sehr ähnliches Verhalten 
wie das, welches $M$ ü n z er z. B. kürzlich bei Kochsalzinfusion gefunden hat. Es findet niemals sofort eine Eintrocknung des Blutes statt (trotzdem dasselbe nach $\mathrm{Cz}$ erny eine erhöhte Viscosität zeigt), sondern eine starke Wasserströmung aus den Geweben in das Blut hinein tritt zu Tage, welehe den Wassergehalt desselben auf seiner ursprünglichen Höhe zu erhalten bestrebt ist. Noch schneller jedoch als bei den krystalloiden Körpern findet sich bei den colloiden der Zeitpunkt, wo diese Ausgleichung nicht mehr möglich wird, und wo der Wassergehalt des Blutes in merklicher Weise und sogar noch viel stärker als bei den eigentliehen Salzen zunimmt. Dies wird aus den beifolgenden Protokollen ersichtlich sein.

Versuch V.

Kaninchen, 1800 g. 3 g Urethan. Blaseneaniile.

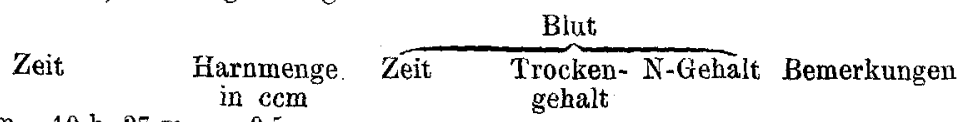

10 h. $17 \mathrm{~m} .-10$ h. $27 \mathrm{~m}$.

10 h. $27 \mathrm{~m} .-10$ h. $37 \mathrm{~m}$.

10 h. $37 \mathrm{~m} .-10 \mathrm{~h} .47 \mathrm{~m}$.

0,5
0,8
0,9

$10 \mathrm{~h}, 22 \mathrm{~m} . \quad 18,64$ Proc. -

Von $10 \mathrm{~h} .46 \mathrm{~m}$. alle Minuten $2 \mathrm{ccm}$ einer 3 procent. Leimlösung intravenös.

10 h. 47 m. -10 h. 57 m. $\quad 1,2 \quad 10$ h. 51 m. 18,44 Proc. 2,902

10 h. $57 \mathrm{~m} .-11$ h. $07 \mathrm{~m} . \quad 1,6 \quad 11$ h. $04 \mathrm{~m} .17,66=2,777$

11 h. 07 m. -11 h. 17 m. $\quad 3,95 \quad 11$ h. 10 m. Harn

11 h. $17 \mathrm{~m} .-11$ h. $27 \mathrm{~m} . \quad 6,55 \quad 11$ h. $21 \mathrm{~m} . \quad 16,52=2,509$ Harn mehrblutig.

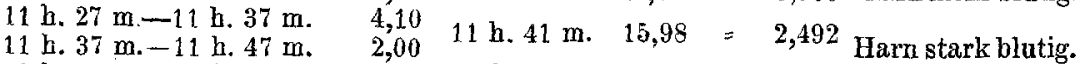

11 h. $47 \mathrm{~m} .-11$ h. $57 \mathrm{~m} . \quad 0,9 \quad 11$ h. $56 \mathrm{~m} . \quad 13,47=2,057$

11. h. 57 m. -12 h. 02 m. ea. 0,2 12 h. 04 m. $11,29=1,707$

12 h. $04 \mathrm{~m}$. Exitus. Der Harn nach der Infusion war leimbaltig, gelatinirend.

Die ausgesprochenere Einwirkung der colloidalen Körper auf den Trockengehalt des Blutes lässt uns auch die folgenden Versuche verständlich erscheinen, welche zeigen, dass nach Infusion grösserer Quantitäten colloidaler Lösungen in das. Blut (und auch für Salzlösungen kann man Aehnliches feststellen) die diuretische Wirkung des Coffeïns vermisst werden kann. Während bei kleineren Dosen colloider Körper die Coffeïndiurese deutlich zu Tage tritt (Versuch VI), bleibt sie (wie Versuch VII zeigt) bei grösseren Dosen ganz oder fast ganz ans. Auf die Bedeutung dieses Befundes für die Theorie der Coffënwirkung soll im Zusammenhang mit anderen Thatsachen anderen Ortes eingegangen werden.

\section{Versuch VI.}

Kaninchen, $2400 \mathrm{~g}$. Narkose durch 2,5 g Urethan. Blasencanüle. Das Thier hatte reichlich Rüben gefressen. 
Zeit

10 h. 5 m.- -10 h. 15 m. 10 h. $15 \mathrm{~m} .-10$ h. $25 \mathrm{~m}$. 10 h. $25 \mathrm{~m} .-10$ h. $35 \mathrm{~m}$. 10 h. 35 m. -10 h. 45 m. Von 10 h. 35 m. -10 h. 45 m.

10 h. 45 m. -10 h. 55 m. Von 10 b. 45 m. - 10 h. $55 \mathrm{~m}$.

10 h. $55 \mathrm{~m} .-11$ h. $05 \mathrm{~m}$. 11 h. $05 \mathrm{~m} .-11$ h. $15 \mathrm{~m}$. 11 h. 15 m. -11 h. $25 \mathrm{~m}$. $11 \mathrm{~h} .25 \mathrm{~m} .-11 \mathrm{~h} .35 \mathrm{~m}$. Von 11 h. 34 m. -11 h. 35 m.

11 h. $35 \mathrm{~m} .-11$ h. $45 \mathrm{~m}$. 11 h. $45 \mathrm{~m} .-11$ h. $55 \mathrm{~m}$.
Harnmenge

$3,7 \mathrm{ccm}$

$3,1=$

$4,2=$

$3,9=$

werden $6 \mathrm{~g}$ Gummi arabicum (in $25 \mathrm{ccm}$ Wasser) intravenös injicirt.

$5,1 \mathrm{ccm}$

werden weitere $2 \mathrm{~g}$ Gummi arabicum (in $10 \mathrm{cem}$ Wasser) intravenös injicirt.
$6,9 \mathrm{cem}$
$8,5=$
$8,3=$
$7,6=$

werden $0,04 \mathrm{~g}$ Coffeïn (in $4 \mathrm{ccm}$ Wasser) intravenös injicirt.

27 eem

$14,5=$

Versuch VII.

Kaninchen hatte 36 Stunden gehungert, die Harnmengen waren in je 10 Minuten $0,05-0,15$ vor der Injection von Gummi arabicum (5 g), und ebenso nach derselben, Coffeinnwirkung war fast gar nicht zu erkennen, die Harnmengen betrugen nie mehr als $0,2 \mathrm{ccm}$ pro 10 Minuten. Das Blut enthielt vor der Injection der Gummilösung 76,58 Proc. Wasser, am Anfang der Injection, $10 \mathrm{~h} .37 \mathrm{~m}$. (die Injection fand von $10 \mathrm{~h} .33 \mathrm{~m}$. bis 10 h. 50 m. statt) 78,48 Proc., 5 Minuten nach der Injection 92,08 Proc., 20 Minuten nach derselben noch 90,59 Proc. Diese Zahlen werden bestätigt durch die entsprechenden Stickstoffzahlen: 1,357 Proc., 1,256 Proc., 0,8213 Proe., 0,8215 Proc.

Die allgemeinen Erscheinungen, welche nach Infusion colloidaler Lösungen zu beobachten sind, unterscheiden sich jedoch nicht unwesentlich von den nach reiner Salzwirkung beobachteten. Die anatomischen Befunde hat $\mathrm{Cz}$ erny schon beschrieben; anschliessend an ihn will ich nur bemerken, dass mir an den zur quantitativen Bestimmung entnommenen Blutproben öfter eine abnorme Schwergerinnbarkeit auffiel. Jodoch war dieser Befund kein so constanter, dass etwa an eine Analogie mit der bekannten Albumosenwirkung gedaeht werden könnte; auch bei dieser Wirkung der Albumosen handelt es sich vermuthlich um eine specifische Reaction, die nicht anderen colloidalen Körpern eigenthümlich ist.

In Bezug auf die allgemeinen Wirkungen treten aber namentlich betreffend Blutdruck und Nervenmuskelsystem wesentliche Differenzen auf. Während für Athmung und Puls bei vorsichtig geleiteter Infusion nichts Wesentliches zu beobachten ist, zeigt der 
Blutdruck von Beginn der Injection an eine Steiger ung. Dieselbe wird sogar allmählich sebr beträchtlich, und ich babe Steigerungen gesehen bis über das Anderthalbfache der ursprünglichen Höhe. Erst kurz vor dem Tode, wenn der Nachlass der Diurese gewissermaassen die Insufficienz der Körpergewebe für die Ausgleichung der osmotischen Verhältnisse anzeigt, sinkt der Blutdruck rapid.

Noch auffallender sind die Erseheinungen am Nervenmuskelsystem. Bekanntlich beobachtet man nach der Infusion von Salzen gesteigerte Reflexerregbarkeit, welche mit fibrillären Zuckungen, Muskelkrämpfen und allgemeinen clonisch-tonisehen Streckkrämpfen einhergeht. Dieses Bild der Vergiftung fehlt bei den "Colloidthieren" vollkommen, dieselben liegen da in tiefster Narkose, vergleichbar, wie mir Prof. Hofmeister sagte, tief abgekühlten Thieren ${ }^{1)}$.

Die Reflexerregbarkeit derselben ist fast vollkommen erlosehen. Dieselben zeigen auch gegentuber Giften, welche sonst Tetanus hervorrufen, eine vollkommene Toleranz. Ich habe gelegentlich den "Colloidthieren" grössere Dosen Coffeïn geben können, obne die sonst so markanten Wirkungen auf das Muskelsystem (Tetanus) beobachten zu können. Auch auf Blutdruck und Herzthätigkeit war bei ihnen die Coffeïnwirkung eine minimale. Es verdient wohl hervorgehoben zu werden, dass diese Thiere, bei denen die Coffeînwirkungen so wenig ausgesprochen waren, auch relativ sehr hohe Dosen des Giftes vertrugen, bis zu $0,65 \mathrm{~g}$, ohne zu sterben. Wie man sieht, tragen die die Giftwirkung begleitenden Symptome, namentlich der Muskelkrampf, auch ihrerseits zum Tode bei, wenn sie auch nicht die eigentliche Ursache des Todes sind.

Diese wesentliche Differenz, welche also zwischen den "Salzund Colloidthieren" besteht, lässt sich wohl ungezwungen in der folgenden Weise erklären: Dass die Erseheinungen nach Salzwirkung vom Gehirn ausgelöst werden, ist leicht, namentlich durch Curarisirung zu beweisen: die in das Gefässsystem injicirten Salze diffundiren in die Gewebe und rufen dort, d. h. vom Gehirn aus, die bezeichneten Erseheinungen hervor. Die colloiden Körper diffundiren bekanntlich sehr schlecht: Infolge dessen treten hier die osmotischen Erscheinungen am Gefässsystem in den Vordergrund, und die Veränderungen im Blut („Eindiekung“") bilden das hervorstechendste und bedeutungsvollste Symptom.

1) Vgl. die Arbeit von Winternitz, Archiv f. experiment. Pathol. u. Pharmakol. Bd. XXXIII, S. 286, 1894. 
Unter den Salzen kann man mit den Colloiden am ehesten noch das Natriumsulfat rergleichen. Denn nach den Untersuchungen von Münzer zeigt dieses nicht nur die relativ höchste Dosis $(4,47 \mathrm{~g}$ pro Kilogramm) als tödtliche für den Thierkörper, sondern es wird auch procentisch in der grössten Quantität im Harn wieder ausgegeschieden, d. h. es vertheilt sich am wenigsten im Organismus. Auf dieses analoge Verhalten hat auch Schmiedeberg in seiner Arzneimittellehre hingewiesen bei Besprechung der Gruppe des Glaubersalzes 1\%. Wie die Ursache der abfürenden Wirkung der Salze dieser Gruppe unzweifelbaft darin zu suchen ist, dass sie im Magen- und Darmkanal im Gegensatz zu denen der Kochsalzgruppe nur langsam und in geringer Menge resorbirt werden, das Wasser in Form ihrer Lösungen gebunden enthalten und seine Aufsaugung verhindern, so wirken auch durch die gleichen Eigenschaften in Wasser leicht lösliche ungiftige organische Stoffe. Hierher gehören also wohl auch die von mir untersuchten colloiden Stoffe, die, wenn sie auch wegen ihres hohen Moleculargewichtes in gelöstem Zustande keine erheblichen molecular-physikalischen Wirkungen entfalten, dieselben doeh im Organismus wegen ihrer geringen Diffusibilität und Resorptionsfähigkeit zeigen können.

Was endlich die anfangs erörterte Wirkung der Albumosen anlangt, so zeigen unsere Untersuchungen, dass an derselben ihre colloidale Beschaffenheit nicht betheiligt ist. Offenbar bandelt es sich vielmehr, wie dies für die gerinnungshemmende Wirkung auch aus einer grossen Reihe anderer Untersuchungen hervorgeht, um physiologisehe (nicht molecularphysikalische) sogenannte specifische Wirkungen dieser Körperklassen. Inwieweit hierbei etwa Einfliusse bestimmter chemischer Constitution oder, was oft vermuthet wurde, fermentartige Beimengungen maassgebend sind, soll weiteren Untersuchungen überlassen bleiben.

1) Grundriss der Arzneimittellehre, S. 264. 